\title{
Effects of Exercise Training in Hypoxia Versus Normoxia on Vascular Health: Comments on Clinical Importance
}

\author{
Mohadeseh Sani $^{1} \cdot$ Erfan Ayubi $^{2,3} \cdot$ Salman Khazaei $^{4} \cdot$ Kamyar Mansori $^{5,6}$
}

Published online: 30 August 2016

(c) Springer International Publishing Switzerland 2016

Dear Editor,

We read with great interest the paper by Montero and Lundby published in Sports Medicine in June 2016 [1]. The authors conducted a systematic review and meta-analysis to clarify the impact on vascular adaptations of exercise training performed in hypoxic versus normoxic conditions. The results showed that the standardized mean differences (SMDs) with $95 \%$ confidence intervals (CI) for skeletal muscle capillarization and vascular dilator function in exercise training performed in hypoxia versus normoxia were 0.40 (95\% CI $0.10-0.70)$ and 0.67 (95\% CI $0.17-1.18$ ), respectively.

Although the statistical methodology used was correct and the data were interesting, some methodological and statistical issues should be considered. Most importantly, while SMDs of 0.40 and 0.67 for mean skeletal muscle

Erfan Ayubi

aubi65@gmail.com

1 School of Medicine, Zabol University of Medical Sciences, Zabol, Iran

2 Department of Epidemiology, School of Public Health, Shahid Beheshti University of Medical Sciences, Tehran 19836, Iran

3 Department of Epidemiology and Biostatistics, School of Public Health, Tehran University of Medical Sciences, Tehran, Iran

4 Department of Epidemiology, School of Public Health, Hamadan University of Medical Sciences, Hamadan, Iran

5 Social Determinants of Health Research Center, Kurdistan University of Medical Sciences, Sanandaj, Iran

6 Department of Epidemiology, School of Public Health, Iran University of Medical Sciences, Tehran, Iran capillarization and vascular dilator function were statistically significant, clinically these differences were unimportant. It is crucial to emphasize that clinical importance and statistical significance are different concepts, and that clinical importance carries more weight than statistical significance. A significant $p$ value is likely to be obtained as a result of a larger sample size, lower standard deviation, and greater mean difference [2]. Data pooling in metaanalyses results in a larger sample size and can easily lead to a significant $p$ value.

The take-home message for readers is that they should exercise clinical judgment when interpreting data.

\section{Compliance with ethical standards}

Conflict of interest Mohadeseh Sani, Erfan Ayubi, Salman Khazaei, and Kamyar Mansori declare that they have no conflicts of interest relevant to the content of this letter.

\section{References}

1. Montero D, Lundby C. Effects of exercise training in hypoxia versus normoxia on vascular health. Sports Med. 2016. doi:10. 1007/s40279-016-0570-5 [Epub 2016 Jun 10].

2. Fletcher RH, Fletcher SW, Fletcher GS. Clinical epidemiology: the essentials. Philadelphia: Lippincott Williams \& Wilkins; 2012. 